\title{
Optisches Lokalisierungssystem für autarke Sensorknoten in industriellen loT Anwendungen
}

\author{
Leander B. Hörmann ${ }^{1}$, Markus Pichler-Scheder ${ }^{1}$, Peter Priller ${ }^{2}$, Hans-Peter Bernhard ${ }^{3}$, \\ Andreas Springer ${ }^{3}$ \\ ${ }^{1}$ Linz Center of Mechatronics GmbH, Area: Sensors and Communication, \\ Altenberger Straße 69, 4040 Linz, Österreich \\ ${ }^{2}$ AVL List GmbH, ITS Global Research \& Technology, \\ Hans-List-Platz 1, 8020 Graz, Österreich \\ ${ }^{3}$ Institut für Nachrichtentechnik und Hochfrequenzsysteme, Johannes Kepler Universität Linz, Alten- \\ berger Straße 69, 4040 Linz, Österreich
}

\begin{abstract}
Zusammenfassung
Zur Überwachung von industriellen Prozessen und zur laufenden Messdatenerfassungen von Industrieanlagen und -Geräten werden immer mehr und mehr Sensoren benötigt. Diese Entwicklung wird vor allem durch industrielle Internet-of-Things (IOT) Anwendungen gleichzeitig ermöglicht und vorangetrieben. Die Verkabelung stellt einen erheblichen Aufwand und potenzielle Fehlerquelle dar. Daher sind autarke Sensorknoten, die ihren Leistungsbedarf mittels sogenanntem Energy Harvesting aus ihrer Umgebung decken und eine drahtlose Kommunikationstechnologie zur Datenübertragung verwenden, oft von Vorteil. Die Sensorknoten bilden ein drahtloses Sensornetzwerk, um die Daten effizient an den Zielort zu übertragen. Weiters sind autarke Sensorknoten in bestimmten Situationen notwendig, da dort eine kabelgebundene Messung nicht möglich ist. Für Anwendungen, bei welchen sich das Messobjekt oft ändert und daher die Sensoren neu angebracht werden müssen, verringert man durch die Verwendung von autarken Sensorknoten die Anzahl von Kabeln zu einer zentralen Messdatenerfassung. Die Zuordnung vom jeweiligen Sensorknoten zu einer bestimmten Messgröße ist jedoch aufwendig und muss meist händisch vorgenommen werden. Um dies zu vereinfachen präsentiert dieser Beitrag ein optisches Lokalisierungssystem für autarke Sensorknoten in industriellen loT Anwendungen. Die Sensorknoten sind mit einer schaltbaren Lichtquelle ausgestattet, welche eine kodierte Blinksequenz erzeugen. Diese Blinksequenz wird von mindesten zwei Kameras erfasst und zur Identifizierung und gleichzeitigen Lokalisierung der Sensorknoten verwendet. Dadurch kann eine teilautonome Zuordnung eines Sensorknotens zu einer bestimmten Messgröße erfolgen.
\end{abstract}

Keywords: Optische Lokalisierung, autarke Sensorknoten, Sensornetzwerk, industrielles loT.

\section{Einleitung}

Autarke Sensorknoten werden immer häufiger zur Erfassung industrieller Messgrößen verwendet. Einerseits wird diese Entwicklung durch die technologische Verfügbarkeit und Verbesserung von kostengünstigen und energieeffizienten Transceiver-Schaltungen und Erhöhung der Rechenleistung integrierter Schaltungen ermöglicht. Andererseits sind zur weiteren Optimierung technischer Prozesse zusätzliche Messdaten notwendig und werden daher von der Anwendung selbst gefordert. Um diese zusätzlichen Messdaten kosteneffektiv erfassen und meist an eine zentrale Einheit übertragen zu können, werden die Sensorknoten zu sogenannte drahtlose Sensornetzwerke („Wireless Sensor Networks“, WSN) zusammengefasst. Die Daten werden drahtlos unter
Einhaltung eines Kommunikationsprotokolls zu einer Datensenke übertragen. Die Sensorknoten selbst erfassen die physikalische Messgröße und vorverarbeiten die Messdaten. Sensorknoten und Sensornetzwerke sind essenzielle Bestandteile des sogenannten industriellen Internet der Dinge („Industrial Internet-ofThings", lloT).

Durch die drahtlose Kommunikation wird eine erhebliche Zahl an Messleitungen eingespart. Um nun nicht auf kabelgebunden Energieversorgung angewiesen sein zu müssen, sind grundsätzlich zwei verschieden Prinzipien möglich [2]. Erstens kann die für die gesamte Betriebszeit des Sensorknotens nötige Energie bereits bei Installation in Form von Batterien mit eingebracht werden. Die maximale Betriebszeit ergibt sich im Wesentlich aus der durchschnittlichen Leistungsaufnahme des 
Sensorknotens und der Selbstentladung der Batterien. Für bestimmte Anwendungsfälle kann dies die kostengünstigste Lösung darstellen, vor allem dann, wenn die Sensorknoten zu Wartungs- und Kalibrierungszwecken regelmäßig getauscht werden müssen.

Zweitens kann der Sensorknoten mittels der in der Umgebung verfügbaren Leistung versorgt werden. Dabei wird durch sogenanntes „Energy Harvesting" diese Umgebungsleistung in elektrische Leistung umgewandelt. Dazu können zum Beispiel Thermogeneratoren oder Solarzellen eingesetzt werden. Die verfügbare Energie aus der Umgebung ist im Allgemeinen unbegrenzt, es kann jedoch meist nur eine geringe elektrische Leistung je nach Anwendungsort erzielt werden [3]. Weiters schwankt die umwandelbare Leistung mit der Zeit und ist im Detail nicht vorhersagbar [4]. Daher muss Energie in Superkondensatoren oder wiederaufladbaren Batterien zwischengespeichert werden, um einen kontinuierlichen Betrieb des Sensorknotens gewährleisten zu können.

Lokalisierungssysteme werden mit zunehmender Anzahl von Sensorknoten immer wichtiger. Eine automatisierte Erfassung der Position der Sensorknoten kann zur schnellen und zuverlässigeren Konfiguration von Sensornetzwerken verwendet werden. Vor allem bei statischen Anwendungen, bei welchen sich die Position der Sensoren bezogen auf das Messobjekts nicht ändert, ist dies sinnvoll. Optische Methoden haben für solche Anwendungen eine gute Genauigkeit. Kameras können die Sensorknoten mittels an innen angebrachten schaltbaren Lichtquellen erfassen. Wenn ein Sensor von mindestens zwei Kameras erfasst wird und die Position und Orientierung derer bekannt ist, kann daraus die Position des Sensorknotens berechnet werden. Bisher stellte der Energieverbrauch der schaltbaren Lichtquelle ein erhebliches Problem dar. Dieser Beitrag präsentiert ein optisches Lokalisie- rungssystem, welches einerseits eine hohe Genauigkeit im unteren Zentimeterbereich erreicht und andererseits den Energieverbrauch der schaltbaren Lichtquelle der Sensorknoten reduziert.

Der folgenden Abschnitte beschreiben das optische Lokalisierungssystem, das verwendete Kommunikationsprotokoll und die optimale Blinksequenz. Danach werden Messergebnisse und die erreichte Messgenauigkeit dargestellt. Schlussendlich fasst der letzte Abschnitt den Beitrag zusammen und gibt einen Ausblick über künftige Entwicklungen.

\section{Optisches Lokalisierungssystem}

Für die optische Lokalisierung der Sensorknoten in 3 Dimensionen werden wie in Abb. 1 dargestellt rund um den Messbereich Kameras (K) installiert und so platziert, dass sich jeder Sensorknoten (S) im Sichtbereich von mindestens zwei Kameras befindet. Um allenfalls Bereiche im Testaufbau abdecken zu können, die ansonsten von Teilen des Messaufbaus, Kabeln, etc. verdeckt wären, kann die Anzahl an Kameras gemäß den Erfordernissen im jeweiligen Szenario erhöht werden. Die Sensorknoten werden mit einer schaltbaren Lichtquelle, im Speziellen mit einer hellen Leuchtdiode (LED) ausgestattet, die in einem transparenten Teil der Sensorgehäuse so angebracht ist, dass sie aus möglichst vielen Raumrichtungen direkt sichtbar ist. Abb. 2 zeigt die aktuelle Ausgestaltung eines solchen Sensorknotens. Für die Datenübertragung sind die Kameras über Ethernet mit einer zentralen Steuerungs- und Signalverarbeitungs-Einheit verbunden, die Triggerung der Blinksequenzen in den Sensoren und der Aufzeichnung von Kamerabildern erfolgt über das im nachfolgenden Abschnitt beschriebene Kommunikationsprotokoll. 


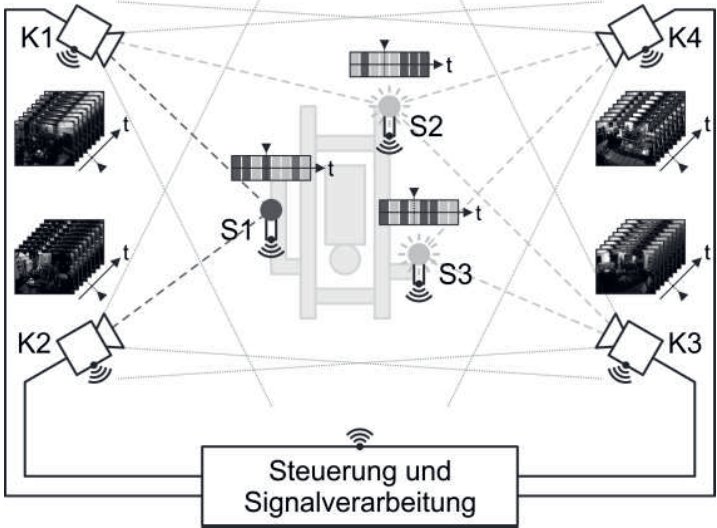

Abb. 1: Schematischer Aufbau der optischen Lokalisierung mit 4 Kameras (K1-K4) und 3 Sensoren (S1-S3) mit Darstellung der zugehörigen Blinksequenz.

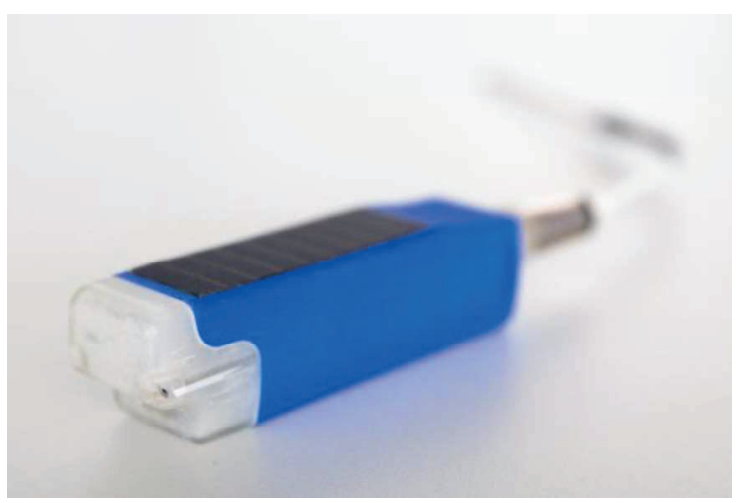

Abb. 2: Sensorknoten mit transparentem Gehäuseteil für optische Lokalisierung.

Zur Lokalisierung wird durch jede Kamera zunächst eine Sequenz von Bildern aufgezeichnet. Synchron zu den Aufnahmezeitpunkten wird durch Ein- und Ausschalten der LEDs in den Sensoren eine für jeden Knoten individuell codierte Blinksequenz durchlaufen, anhand derer die einzelnen Sensoren schließlich voneinander unterschieden werden können. Die Länge der Blinksequenz bestimmt den Umfang des Coderaums und damit die maximale Anzahl der lokalisierbaren Sensorknoten; gleichzeitig erhöht aber eine Verlängerung der Blinksequenz auch den Energieverbrauch für die Lokalisierung. Eine Analyse zur optimalen Blinksequenz ist in einem späteren Abschnitt gezeigt.

Während der Aufzeichnung sollte nach Möglichkeit ein statisches Szenario herrschen, sich in den Kamerabildern bis auf die unterschiedlichen Leuchtzustände der LEDs also möglichst wenig ändern. Das erleichtert die Detektion auch nur schwach leuchtender oder teilweise verdeckter LEDs in den Kamerabildern und verringert die Wahrscheinlichkeit von Fehlde-

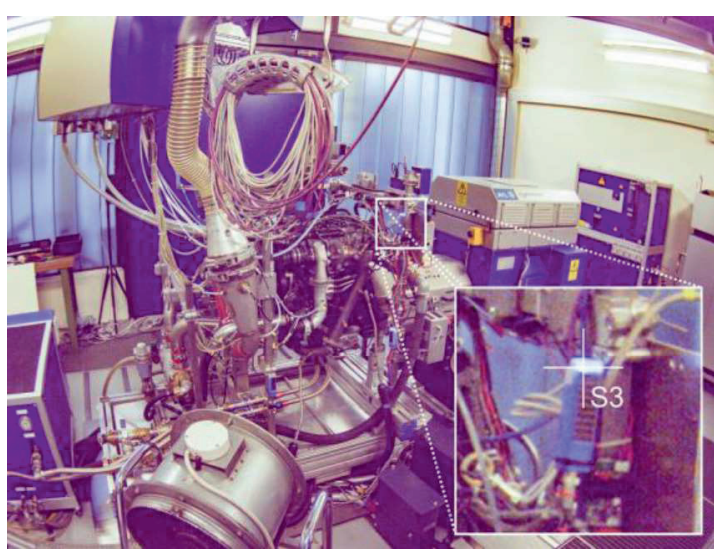

(a)

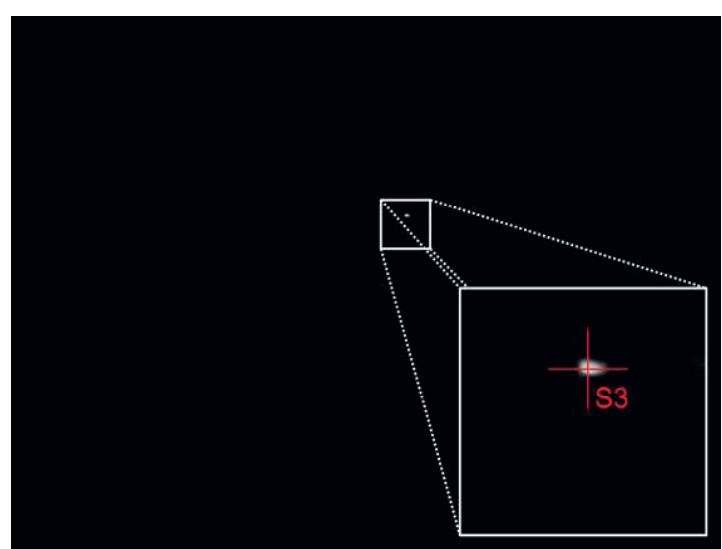

(b)

Abb. 3: Exemplarisches (a) Kamerabild und (b) zugehöriges Detektionsbild für die Detektion von Sensor S3.

tektionen. In den typischen Szenarien, in denen diese Methode zum Einsatz kommen soll, ist dies in der Regel einfach sicherzustellen. Verbleibende unvermeidliche unerwünschte Änderungen in den Kamerabildern etwa durch Bildrauschen, Vibrationen, Beleuchtungsänderung etc. können durch robuste Schätzmethoden in der Signalverarbeitung kompensiert werden.

In den von den Kameras aufgenommenen Bildsequenzen werden nun zunächst die einzelnen installierten Sensoren anhand der bekannten Blinksequenzen detektiert und in Bildkoordinaten jeder Kamera lokalisiert. Dabei sind zum einen die Sensorknoten voneinander zu unterscheiden, aber auch zu entscheiden, ob ein bestimmter Sensorknoten von einer Kameraposition aus überhaupt sichtbar ist. Hierzu wird aus den Einzelbildern jeder Kamera durch Addition bzw. Subtraktion gemäß dem Code jedes installierten Sensorknotens ein Detektionsbild errechnet, in dem anschließend das Maximum gesucht wird. Ein exemplarisches Kamerabild und ein zugehöriges Detektionsbild für Sensor S3 sind in Abb. 3 dargestellt. 

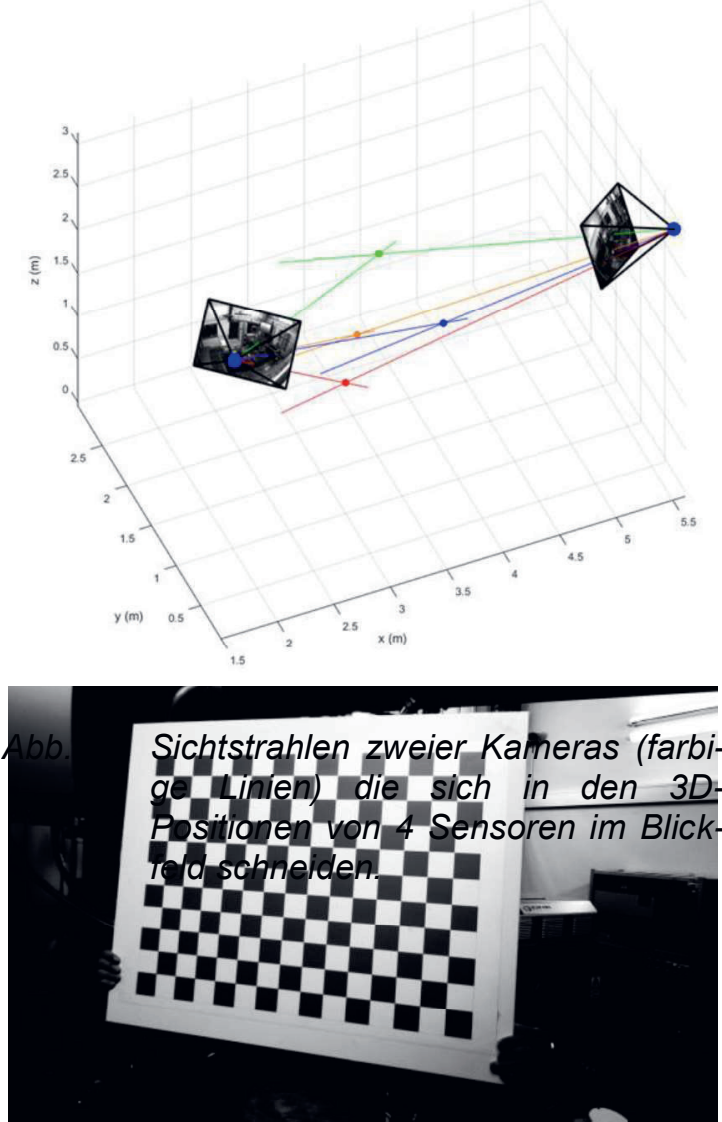

(b)

Abb. 4: Kamerabild des zur Kalibrierung verwendeten

(a) vor und (b) nach Entzerrung.

Nach der Detektion stehen für jede Kamera die Koordinaten aller sichtbaren Sensoren in Kamerakoordinaten zur Verfügung. Für die Umrechnung in Raumkoordinaten ist eine Kalibration der Kameras erforderlich, um unvermeidliche Verzerrungen der Objektive zu entfernen. Eine gängige Methode hierfür ist die Aufnahme einer mit einem Schachbrettmuster versehenen Tafel aus verschiedenen Blickwinkeln, woraus die Parameter der Kameraoptik berechnet und die aufgenommenen Bilder entzerrt werden können, wie exemplarisch in Abb. 4 dargestellt. Sofern Position und Orientierung der Kameras im Raum bekannt sind, lässt sich das Bild jeder detektierten Leuchtdiode nun in einen Sichtstrahl im Raum umrechnen. Die Sichtstrahlen verlassen die Kameras unter durch die zuvor ermittelten Bildkoordinaten der Leuchtdioden gegebenen Winkel, weshalb es sich hierbei um ein „Angle-of-Arrival“Messverfahren handelt. Wie in Abb. 5 schematisch dargestellt, schneiden sich die von verschiedenen Kameras ausgehenden Sichtstrahlen im Idealfall an der 3D-Position des entsprechenden Sensors. In der Realität schneiden sich die Strahlen wegen unvermeidlicher

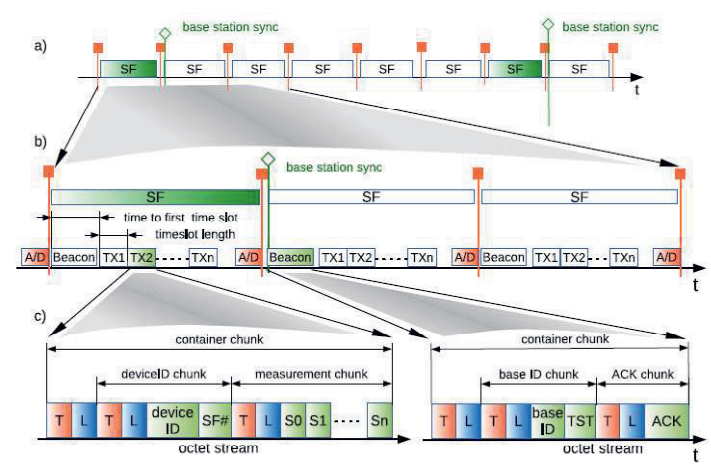

Abb. 6: a) Superframe-Taktstruktur, b) Superframe-Aufbau und c) Datenstruktur des EPhESOS Kommunikationsprotokolls.

Messfehler, nicht vollständig kompensierter optischer Verzerrungen sowie Unsicherheiten in Kamerapositionen und -orientierungen nicht in einem Punkt, sondern verlaufen windschief. Als Schätzergebnis wird deshalb derjenige Punkt ermittelt, der den quadratischen Abstand zu allen Sichtstrahlen minimiert.

\section{Kommunikationsprotokoll}

Im Folgenden wird das verwendete Kommunikationsprotokoll EPhESOS beschrieben. Dieses Protokoll ist speziell für drahtlose Sensornetzwerke entwickelt worden, die eine möglichst geringe durchschnittliche Leistungsaufnahme sowie eine geringe Latenzzeit benötigen. Der durchschnittliche Leistungsverbrauch wird durch die Verwendung eines TDMAartigen Protokolls minimiert. Dazu müssen die Sendezyklen exakt aufeinander abgestimmt und die Sensorknoten untereinander genau synchronisiert sein. Dadurch kann die Sendeund Empfangszeiten der einzelnen Sensorknoten minimiert werden.

Die Frame-Struktur des EPhESOSKommunikationsprotokolls ist in Abb. 6 gezeigt. Es basiert auf einem TDMA MAC Verfahren, welches Zeitschlitze innerhalb eines Superframes (SF) vorgibt (Abb. 6a). Jeder SF besteht aus einem Zeitfenster für einen Beacon zur Downlink-(DL)-Kommunikation und einem Zeitfenster für jeden Sensorknoten zur Uplink-(UL)-Kommunikation (Abb. 6b). Je nach Anwendung können diese Zeitschlitze individual konfiguriert werden. Der Beacon wird zur Übertragung von erstens Synchronisationsnachrichten, zweitens Paketbestätigungsinformationen und drittens Netzwerkbefehle verwendet. Die Datenstruktur sowohl der DL- als auch UL-Pakete werden durch das EPhESOS Datengram strukturiert (Abb. 6c). Eine Struktureinheit besteht aus Type $(T)$, Länge $(L)$ und Wert (V) und wird TLV-Chunk bezeichnet. 
Diese Struktureinheiten können ineinander verschachtelt sein, um Daten noch weiter zu strukturieren. In der Abb. $6 \mathrm{c}$ ist unter anderem der Aufbau eines Beacon-Chunks dargestellt, der die Nummer der aussendenden Basisstation (baselD) und einen Zeitstempel (TST) zur Synchronisation enthält.

Das Kommunikationsprotokoll unterstützt zwei verschiedene Übertragungsmodi, welche im Folgenden kurz erläutert werden: Erstens, der sporadischen Übertragungsmodus EPhESOS$S$ ist für die Netzwerkinbetriebnahme, die Integration neuer Knoten, die Initialsynchronisation, die Knotenkonfiguration und die Wartung zuständig. Bereits in diesem Modus wird die SF-Taktstruktur eingehalten, obwohl noch nicht im jedem SF ein Beacon zu den Knoten gesendet wird. Um einen neuen Knoten in diese Zeitstruktur zu integrieren, sendet dieser zu einem beliebigen Zeitpunkt eine Anfrage an die Basisstation und bekommt eine Antwort mit der Synchronisationsinformation und der Zeitschlitzinformation. Danach wird die SFTaktstruktur auch von diesem Knoten eingehalten. Dadurch kann der drahtlose Sensorknoten, wenn keine Daten übertragen werden sollen, im energiesparenden Schlafmodus verbleiben [5].

Zweitens, der kontinuierlichen Übertragungsmodus EPhESOS-C kann nur aus EPhESOS$S$ erreicht werden, da die Synchronisationsinformation und der Zeitschlitzinformation bereits allen Knoten bekannt sein müssen. In diesem Modus sendet die Basisstation zu Beginn jedes SF einen Beacon, der unter anderem die Empfangsbestätigung von davor gesendeten UL-Paketen enthält. Ein Knoten muss allerdings nicht in jedem SF Daten senden, sondern kann auch Daten aggregieren und diese energiesparend in einem UL-Paket verpacken. Wird ein Paketverlust festgestellt, wird die fehlerhaft übertragenen Daten im folgenden SF erneut gesendet. Die Sensorknoten bleiben zwischen Empfangs- und Sendezeiten im Ruhezustand. Um einen reibungslosen Betrieb zu gewährleisten, ist eine präzise Synchronisation erforderlich, da sonst die Sendezeitpunkte driften würden. Zur Synchronisation im EPhESOS Protokoll wird eine Kombination aus einer Frequenzschätzmethode [6] und einem $\Delta \Sigma$-Ansatz verwendet [7].

Die LEDs der Sensorknoten werden synchron zur SF-Taktstruktur je nach Codevorgabe aktiviert und deaktiviert. Dazu muss sich das Sensornetzwerk im EPhESOS-C-Modus befinden. Zusätzlich werden spezielle Triggerknoten, die an den Kameras angebracht sind, zur Auslösung jener verwendet. Die Codesequenz für diese Triggerknoten ist immer ident und be-
Tab. 1: Parameter zu Berechnung des Energieverbrauchs während eines Lokalisierungsvorganges.

\begin{tabular}{|l|c|}
\hline \multicolumn{1}{|c|}{ Parameter } & Wert \\
\hline Leistungsaufnahme LED & $42 \mathrm{~mW}$ \\
\hline $\begin{array}{l}\text { Durchschn. Leistungsaufnah- } \\
\text { me Comm. \& Sync. }\end{array}$ & $400 \mu \mathrm{W}$ \\
\hline Leistungsaufnahme Ruhe & $30 \mu \mathrm{W}$ \\
\hline $\begin{array}{l}\text { Zeit für Comm. \& Sync. pro } \\
\text { Sekunde }\end{array}$ & $20 \mathrm{~ms}$ \\
\hline Zeit LED aktiv (1 Blink) & $100 \mathrm{~ms}$ \\
\hline Zeit zwischen 2 Blinks & $100 \mathrm{~ms}$ \\
\hline
\end{tabular}

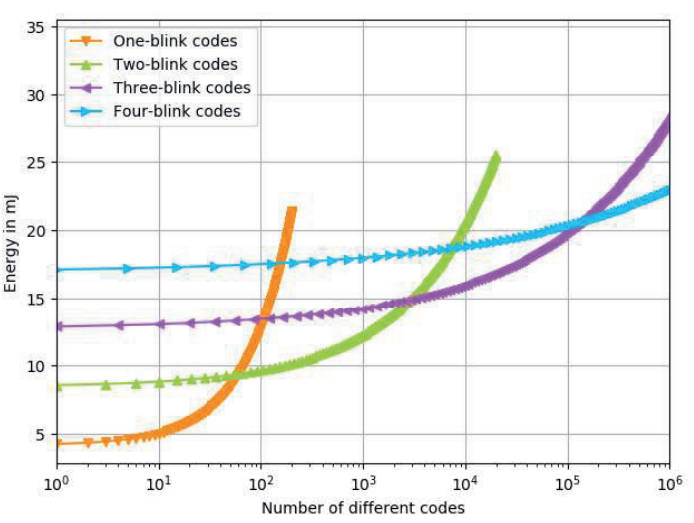

Abb. 7: Energieverbrauch eines Lokalisierungsvorganges pro Sensorknoten über die max. Anzahl an Sensorknoten für ein bis vier LED Aktivierungen.

steht nur aus Aktivierungen, damit jede Kamera in jedem Blinkintervall auslöst.

\section{Optimale Blinksequenz}

Dieser Abschnitt zeigt die Anforderung an die optimale Blinksequenz. Als maßgebliches Optimierungskriterium für drahtlose Sensorknoten wurde der Gesamtenergieverbrauch eines Sensorknotens während eines Lokalisierungsvorganges ausgewählt. Da ein Lokalisierungsvorgang aufgrund der statischen Sensorknotenanordnung nur selten durchgeführt werden muss, ist die Gesamtdauer nicht entscheidend und wird hier nicht berücksichtigt. Dieser Gesamtenergieverbrauch setzt sich aus dem Energieverbrauch der LED zur Signalisierung der Blinksequenz, dem Energieverbrauch zur laufenden Kommunikation und Synchronisation und dem Energieverbrauch in den Ruhezeiten zusammen. Um den Energieverbrauch berechnen zu können wurden folgende Werte herangezogen (siehe Tab. 1).

$\mathrm{Da}$ die LED einen sehr hohen Leistungsverbrauch im Vergleich zu den anderen Komponenten aufweist, ist es sinnvoll diese nur so wenig wie möglich einzuschalten. Im einfachs- 


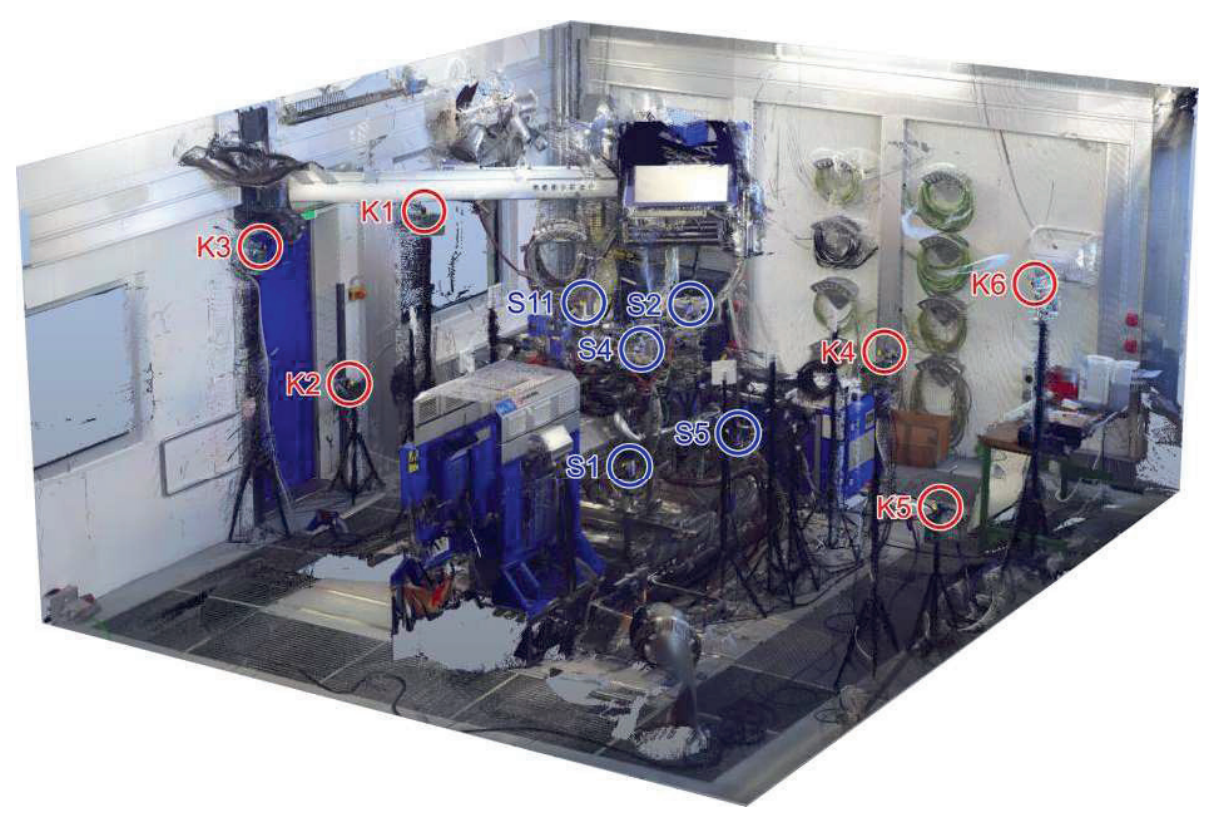

Abb. 8: Ansicht des Messaufbaus aus 3D-Laserscan mit eingezeichneten Kameras (K1-K6) und einigen sichtbaren Sensoren (S).

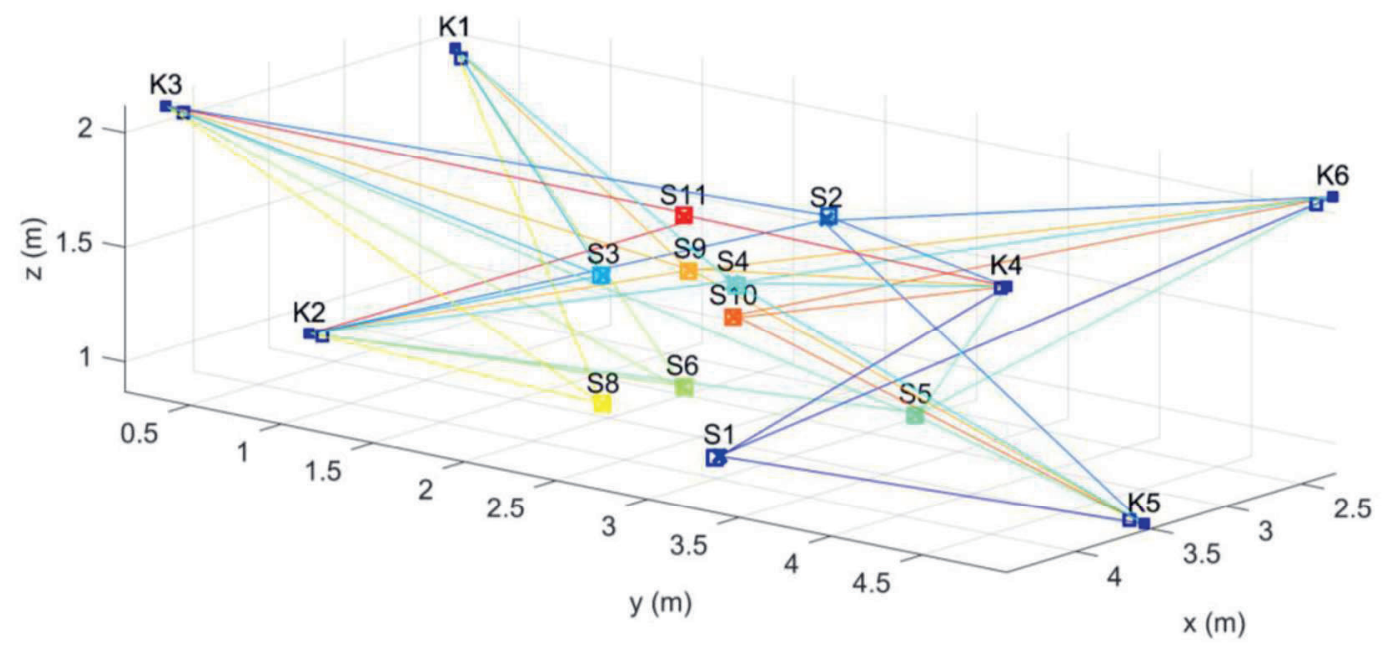

Abb. 9: Dreidimensionale Darstellung von Kamera- (K1-K6) und Sensorpositionen (S). Referenzpositionen aus dem 3D-Laserscan sind als Quadrate ohne Füllung dargestellt, geschätzte Positionen als Kreuze. Für von einer bestimmten Kamera aus sichtbare LEDs sind Sichtstrahlen in der Farbe des Sensors dargestellt.

ten Fall enthält jede Blinksequenz (oder auch Code genannt) nur eine einzelne LEDAktivierung. Dadurch steigt natürlich die Länge der Codes linear mit der Anzahl der teilnehmenden Sensorknoten. Ab einer gewissen Anzahl wird jedoch der Energieverbrauch der LED geringer im Vergleich zum restlichen Energieverbrauch des Knotens. Daher ist es ab einer gewissen Anzahl von teilnehmenden Sensorknoten energetisch besser, pro Code zwei LEDs zu aktivieren. Dieses verhalten ist in Abb. 7 dargestellt. Sie zeigt den Gesamtenergieverbrauch eines Lokalisierungsvorganges eines Sensorknotens für ein bis vier LED
Aktivierungen pro Code in Abhängigkeit von der maximalen Sensorknotenanzahl.

\section{Messergebnisse und Genauigkeit}

Die Lokalisierung wurde in einem Motorprüfstand unter realen Bedingungen getestet. In Abb. 8 ist ein 3D-Laserscan der Testumgebung dargestellt, der auch zur Ermittlung der Referenzpositionen von Sensoren und Kameras verwendet wurde. Im Zentrum des Testbereichs befindet sich ein an einem Metallrahmen montierter Motorblock mit den zugehörigen Zuund Ableitungen von Kraftstoff, Schmiermitteln, Abgasen etc. An diesem Testaufbau wurden 
10 Sensoren (S1-S11, ohne S7) an charakteristischen Positionen montiert. Auf beiden Seiten des Motorblocks wurden jeweils 3 Kameras $(\mathrm{K} 1-\mathrm{K} 6)$ in zwei verschiedenen Höhen platziert, sodass jeder Sensor von mindestens 3 Kameras aus sichtbar war. Unter Verwendung einer Codelänge von 16 wurde eine Serie von Bildern aufgezeichnet und daraus die Sensorpositionen in 3D berechnet. Die Positionen der Kameras wurden hierbei vorgegeben, die Orientierungen wurden ebenso wie die Positionen der Sensoren durch das beschriebene Verfahren geschätzt. In Abb. 9 sind die aus dem Laserscan ermittelten Referenzpositionen (Quadrate), sowie die Sichtstrahlen der Kameras zu den sichtbaren Sensoren, sowie die daraus geschätzten Sensorpositionen (Kreuze) dargestellt. Die räumlichen Distanzen der Schätzungen zu den Referenzpositionen sind in Abb. 10 dargestellt, die Distanzen zwischen den Schätzungen und den einzelnen Sichtstrahlen, die sich in der realen Messsituation nicht perfekt in einem Punkt schneiden in Abb. 11. Man kann erkennen, dass die erzielten Ergebnisse zueinander konsistent sind und die erzielbaren Fehler in einem Bereich von ca. 10-20 mm liegen, der für die Anwendung jedenfalls ausreichend ist.

\section{Zusammenfassung und Ausblick}

Dieser Beitrag präsentiert ein optisches Lokalisierungssystem für autarke Sensorknoten in industriellen loT Anwendungen. Es beschreibt den Anwendungsfall und den Messaufbau. Zusätzlich wird das verwendete Low-Power Kommunikationsprotokoll erklärt und auf die optimale Blinksequenz eingegangen. Dabei zeigt sich, dass je nach Anzahl an Sensorknoten, eine unterschiedliche Anzahl an LED Aktivierungen energieoptimal ist. Weiters sind die Messergebnisse zur Lokalisierung von Sensorknoten an einem Motorprüfstand gezeigt. Dabei ergibt sich ein Fehler der ermittelten Position der Sensorknoten von ca. 5-25 mm.

Zukünftige Entwicklungen zielen auf eine Verbesserung der Lokalisationsgenauigkeit sowie einer automatisierten Zuweisung der optimalen Blinksequenzen zu den jeweiligen Sensorknoten $a b$.

\section{Danksagung}

Diese Arbeit wurde vom LCM - K2 Center im Rahmen des österreichischen COMET-K2Programms und durch die Österreichische Forschungsförderungsgesellschaft $\mathrm{mbH}$ (FFG) unter der Antragsnummer 853456 (FASAN: Flexible Autonome Sensorik in industriellen ANwendungen) unterstützt.

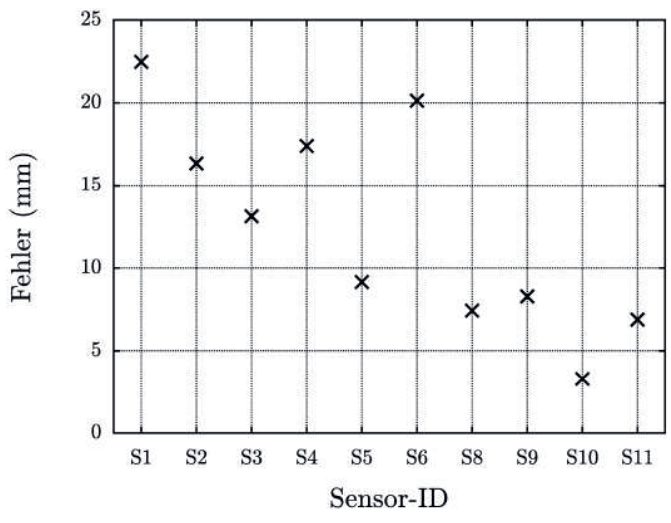

Abb. 10: Lokalisierungsfehler der 10 Sensoren als Abstand der Schätzung von der mit dem Laserscanner vermessenen Referenzposition.

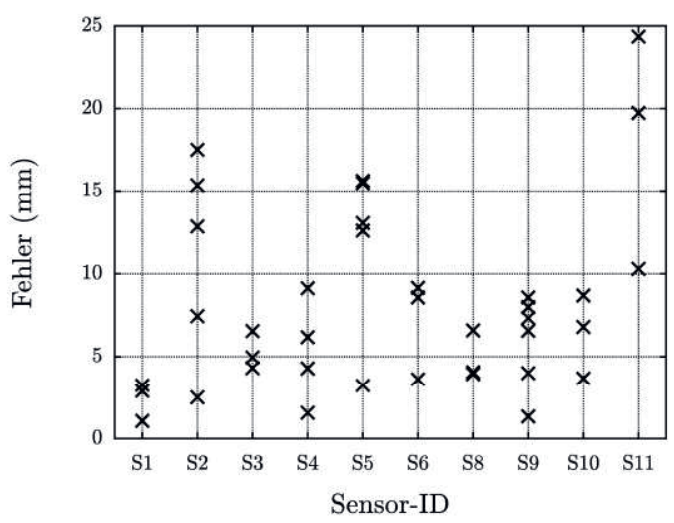

Abb. 11: Lokalisierungsfehler der 10 Sensoren als Abstand der Schätzung von den Sichtstrahlen aller Kameras mit Sichtverbindung zum jeweiligen Sensor.

\section{Literaturnachweis}

[1] M. Bal, "Industrial applications of collaborative wireless sensor networks: A survey," in 2014 IEEE 23rd International Symposium on Industrial Electronics (ISIE), June 2014, 1463-1468; doi: 10.1109/ISIE.2014.6864830

[2] L. B. Hörmann, T. Buchegger, and C. Steger, "Optimizing the Energy Supply of Autonomous Wireless Sensor Nodes," in Microelectronic Systems Symposium (MESS 2014), 2014, 1-6; doi: 10.1109/MESS.2014.7010257

[3] L. B. Hörmann, P. M. Glatz, K. B. Hein, M. Steinberger, C. Steger, and R. Weiss, "Towards an On-Site Characterization of Energy Harvesting Devices for Wireless Sensor Networks," in IEEE International Conference on Pervasive Computing and Communications Workshops (PERCOM Workshops 2012), 2012, 415-418; doi: 10.1109/PerComW.2012.6197523

[4] A. Janek, C. Trummer, C. Steger, R. Weiss, J. Preishuber-Pfluegl, and M. Pistauer, "Simulation based Verification of Energy Storage Ar- 
chitectures for Higher Class Tags Supported by Energy Harvesting Devices," Microprocessors and Microsystems, vol. 32, no. 5-6, 2008, 330339; doi: 10.1016/j.micpro.2008.03.009

[5] H.-P. Bernhard, A. Springer, A. Berger, and P. Priller,"Life cycle of wireless sensor nodes in industrial environments," in 13th IEEE Int. Workshop Factory Commun. Sys., Trondheim, Norway, May 2017; doi:

10.1109/WFCS.2017.7991943

[6] H. P. Bernhard and A. Springer, "Linear complex iterative frequency estimation of sparse and pulse and point processes," in Europ. Sig. Process. Conf. (EUSIPCO), Kos, Greece, Aug. 2017; doi: 10.23919/EUSIPCO.2017.8081380

[7] H.-P. Bernhard, A. Berger, and A. Springer, "Analysis of delta-sigma-synchronization in wireless sensor nodes," in IEEE Int. Conf. Industrial Informatics (INDIN), Cambridge, UK, Jul. 2015, pp. 914-918; doi: 10.1109/INDIN.2015.7281857 